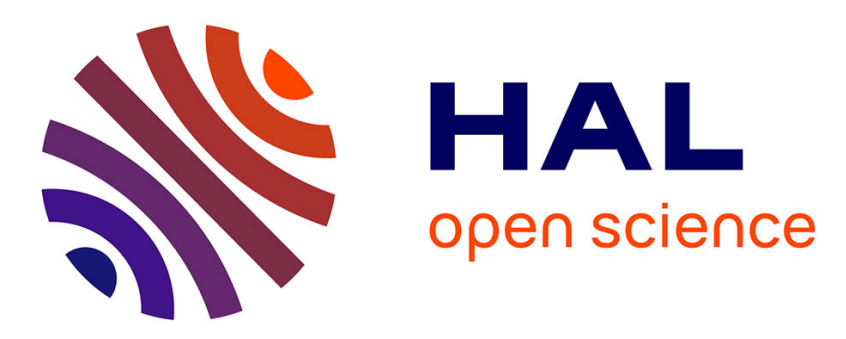

\title{
Order Reduction of Volterra and Volterra-Laguerre Models
}

Noël Tanguy, Mihai Telescu

\section{To cite this version:}

Noël Tanguy, Mihai Telescu. Order Reduction of Volterra and Volterra-Laguerre Models. 21th IEEE Workshop on Signal and Power Integrity (SPI), May 2017, Lake Maggiore (Baveno), Italy. 10.1109/SaPIW.2017.7944014 . hal-01680168

\section{HAL Id: hal-01680168 \\ https://hal.univ-brest.fr/hal-01680168}

Submitted on 10 Jan 2018

HAL is a multi-disciplinary open access archive for the deposit and dissemination of scientific research documents, whether they are published or not. The documents may come from teaching and research institutions in France or abroad, or from public or private research centers.
L'archive ouverte pluridisciplinaire HAL, est destinée au dépôt et à la diffusion de documents scientifiques de niveau recherche, publiés ou non, émanant des établissements d'enseignement et de recherche français ou étrangers, des laboratoires publics ou privés. 


\title{
Order Reduction of Volterra and Volterra-Laguerre Models
}

\author{
Mihai Telescu, Member, IEEE, Noël Tanguy, Member, IEEE \\ Lab-STICC UMR CNRS 6285, Université de Bretagne Occidentale, Université Bretagne Loire, Brest, France \\ mihai.telescu@univ-brest.fr
}

\begin{abstract}
This paper presents a model order reduction method for weakly nonlinear systems. Starting from a Volterra or Volterra-Laguerre model, each independent kernel is efficiently approximated in order to reduce parametric complexity and speed up simulation.
\end{abstract}

Keywords-nonlinear system, Volterra model; VolterraLaguerre model; model order reduction

\section{INTRODUCTION}

Volterra series are one of the best known mathematical frameworks for nonlinear system identification and modeling and have been a compulsory passage for generations of graduate and Ph.D. students. Over the years the early work of Vito Volterra [1] and Wiener [2] was expanded and pursued by countless researchers and has found applications into fields of science ranging from animal and human biology to electromagnetics and control theory. While an exhaustive bibliography is beyond the scope of this paper, it's worth noting a few recent contributions significant both in terms of theoretical novelty and potential applications. The relationship between the Wiener class of nonlinear systems and Volterra models has been proven in [3] and while the immediate application refers to eye-tracking and the characterization of the human smooth pursuit system the results can arguably be reused in other applications. In electronics the relationship between Volterra series and X-parameters has very recently been proven [4]. The potential impact on nonlinear-device macromodeling and simulation can be significant. Finally, it is worth noting the latest developments based on the use of tensor calculus that makes the identification of high order MIMO Volterra systems possible [5].

The present paper uses Volterra models as a starting point and focuses on the compact and accurate approximation of Volterra kernels with the objective of speeding up the simulation of nonlinear models. Traditionally, model order reduction (MOR) was applied to nonlinear systems either following a stage of linearization [6] or within the framework of a trajectory-based piecewise approach [7]. Such approaches are robust and easy to implement, but do have limitations due to the intrinsic parametric complexity or experience difficulties related to the training process. The method presented in this paper follows a different approach. It seeks to preserve all the advantages of Volterra models and all the recent results related to the latter while providing a bonus in terms of computational speed-up. It can be seen as a multilinear generalization of the method described in [8].

\section{VOLTERRA AND VOLTERRA-LAGUERRE MODELS}

Volterra series basically constitute a generalization of the impulse response function and have been used to describe nonlinear systems for over half a century.

$$
y(k)=\sum_{m=1}^{M} \sum_{k_{1}=0}^{k} \ldots \sum_{k_{m}=0}^{k} h_{m}\left(k_{1}, \ldots, k_{m}\right) \prod_{l=1}^{m} x\left(k-k_{l}\right)
$$

With its general and elegant mathematical formulation Volterra series might seem like a straight-forward solution to practically a vast class of nonlinear system identification or behavioral modeling problems. In practice, however Volterra models can very quickly become an unbearable burden due to the huge number of parameters involved. This "curse of complexity" often leads to slow simulations, cumbersome software implementations and quite often so many numerical issues that modelers simply resort to more compact heuristic approaches.

One of the first attempts to solve or at least alleviate the problem of parametric complexity was to use a projection of each kernel on an orthonormal basis leading to

$$
h_{m}\left(k_{1}, \ldots, k_{m}\right)=\sum_{i_{1}=0}^{\infty} \ldots \sum_{i_{m}=0}^{\infty} C_{m, i_{1}, \ldots, i_{m}} \prod_{l=1}^{m} \phi_{m, i_{l}}\left(k_{l}\right) .
$$

This approach is still common today [9]. The usual choice for the orthonormal set is the single-pole Laguerre basis because of its simplicity and effectiveness. Discrete-time Laguerre functions are defined by their z-transform

$$
\Phi_{m, i}(z)=\sqrt{1-a_{m}^{2}} \frac{z}{z-a_{m}}\left(\frac{1-a_{m} z}{z-a_{m}}\right)^{i} .
$$

Let $\psi_{m, i}(k)=\phi_{m, i}(k) * x(k)$. Replacing (2) in (1) allows the input-output system description to be re-written as

$$
y(k)=\sum_{m=1}^{M} \sum_{i_{1}=0}^{\infty} \ldots \sum_{i_{m}=0}^{\infty} C_{m, i_{1}, \ldots, i_{m}} \prod_{l=1}^{m} \psi_{m, i_{l}}(k) .
$$

In practice the infinite series in (4) are truncated. While identification using (4) may have advantages with respect to a direct use of (1) the resulting model may still have a very large 
number of parameters and may be cumbersome to simulate. In any case, it should be noted that if $a_{m}=0$ in (2) for all kernels, (4) reverts to (1), therefore all the derivations that follow hold for both Volterra and Volterra-Laguerre models.

\section{MODEL ORDER REDUCTION METHOD}

\section{A. General framework}

Let the multidimensional z-transform of each discrete-time kernel $h_{m}\left(k_{1}, \ldots, k_{m}\right)$ be denoted as $H_{m}\left(z_{1}, \ldots, z_{m}\right)$. It is convenient, for reasons related to the properties of Laguerre functions, to proceed to a classical change of variables that does not affect the generality of the series. Let $w_{i}=\left(z_{i}-a_{i}\right) /\left(1-a_{i} z_{i}\right)$ with $i=1, \ldots, m$. The series can then be recast in $w$ domain with kernels $\bar{H}_{m}\left(w_{1}, \ldots, w_{m}\right)$ defined by

$$
\bar{H}_{m}\left(w_{1}, \ldots, w_{m}\right)=\prod_{i=1}^{m}\left(\frac{\sqrt{1-a_{i}^{2}} w_{i}}{w_{i}+a_{i}}\right) H_{m}\left(\frac{w_{1}+a_{1}}{1+a_{1} w_{1}}, \ldots, \frac{w_{m}+a_{m}}{1+a_{m} w_{m}}\right)
$$

The inverse transform is given by

$$
H_{m}\left(z_{1}, \ldots, z_{m}\right)=\prod_{i=1}^{m}\left(\frac{\sqrt{1-a_{i}^{2}} z_{i}}{z_{i}-a_{i}}\right) \bar{H}_{m}\left(\frac{z_{1}-a_{1}}{1-a_{1} z_{1}}, \ldots, \frac{z_{m}-a_{m}}{1-a_{m} z_{m}}\right) .
$$

Let us now assume that each kernel allows an approximation in $w$ domain as defined by

$$
\bar{H}_{m}\left(w_{1}, w_{2}, \ldots, w_{m}\right) \cong \frac{N\left(w_{1}, w_{2}, \ldots, w_{m}\right)}{\prod_{i=1}^{m} D_{m, i}\left(w_{i}\right)}
$$

Assumption (6), particularly the product form of the denominator, is instrumental in the order reduction process that follows.

Since this paper is essentially a proof-of-concept targeting a large audience, the authors chose to avoid tensor notations. Model order reduction is first explained for the first order kernel, the generalization is then presented in detail for the second order and inferred for higher orders. To further simplify notations when dealing with the Laguerre coefficients defined by equation (2) index $m$ will be omitted. As the paper addresses the kernels separately there should not be any ambiguity.

\section{B. First order kernel}

The first order kernel of a Volterra system is a linear system and thus (6) is a classic approximation problem defined and solved in [8]. The process begins by defining a set of functions $\Omega_{1}=\left\{G_{0}(w), G_{1}(w), \ldots, G_{R}(w)\right\}$ with the first function being the Volterra kernel itself $G_{0}(w)=\bar{H}_{1}(w)$. Owing to (5) $G_{0}(w)$ may simply be written as

$$
G_{0}(w)=\bar{H}_{1}(w)=\sum_{i=0}^{\infty} C_{i} w^{-i}
$$

One recognizes the Laguerre coefficients $C_{i}$ as they appear in equation (2). All the other functions $G_{j}(w)$ with $j=1 \ldots R$ are obtained by a shift in the spectrum of Laguerre coefficients, i.e.

$$
G_{j}(w)=\sum_{i=0}^{\infty} C_{i+j} w^{-i}
$$

Now let $E_{1}(w)$ be defined by

$$
E_{1}(w)=\sum_{j=0}^{R_{1}} A_{j} G_{j}(w)
$$

with $A_{R_{1}}=1$. At this point $G_{0}(w)$ may be recast as

$$
G_{0}(w)=\bar{H}_{1}(w)=\frac{\sum_{i=0}^{R_{1}-1} C_{i} \sum_{j=1}^{R_{1}-j} A_{j+i} w^{j}+E_{1}(w)}{\sum_{j=0}^{R_{1}} A_{j} w^{j}} .
$$

Coefficients $A_{j}, j=0 \ldots R_{1}-1$, are obtained by solving the simple linear problem given by (9) when seeking to minimize $\left\|E_{1}\right\|$. It follows from (10) that a suboptimal solution of the initial problem (6), for the particular case of $m=1$ is given by

$$
G_{0}(w)=\bar{H}_{1}(w) \cong \frac{\sum_{i=0}^{R_{1}-1} C_{i} \sum_{j=1}^{R_{1}-j} A_{j+i} w^{j}}{\sum_{j=0}^{R_{1}} A_{j} w^{j}}
$$

Note that $R_{1}$ denotes the order to which the first kernel is reduced.

\section{Second and higher order kernels}

We will next consider the generalization of the algorithm above to the quadratic kernel. The strategy we follow is to perform order reduction along the $w_{1}$ axis and along the $w_{2}$ axis as independently as possible which is in fact the reason for the particular form of the denominator in (6).

The projection of the kernel on a two-dimensional Laguerre basis leads to a matrix of coefficients $C_{i_{1} i_{2}}$ with $i_{1,2}=0,1 \ldots$. Follows an iterative procedure analogous to (8), only this time in two dimensions. Starting with $G_{0,1}\left(w_{1}, w_{2}\right)=G_{0,1}\left(w_{1}, w_{2}\right)=\bar{H}_{2}\left(w_{1}, w_{2}\right)$ the matrix containing the coefficients $C_{i_{1} i_{2}}$ is independently shifted along the axes $w_{1}$ and $w_{2}$ as shown in figure 1. This yields 
$\Omega_{21}=\left\{G_{0,1}\left(w_{1}, w_{2}\right) G_{1,1}\left(w_{1}, w_{2}\right), \ldots, G_{R_{21}, 1}\left(w_{1}, w_{2}\right)\right\}$

and, respectively, $\Omega_{22}=\left\{G_{0,2}\left(w_{1}, w_{2}\right) G_{1,2}\left(w_{1}, w_{2}\right), \ldots, G_{R_{22}, 2}\left(w_{1}, w_{2}\right)\right\}$.

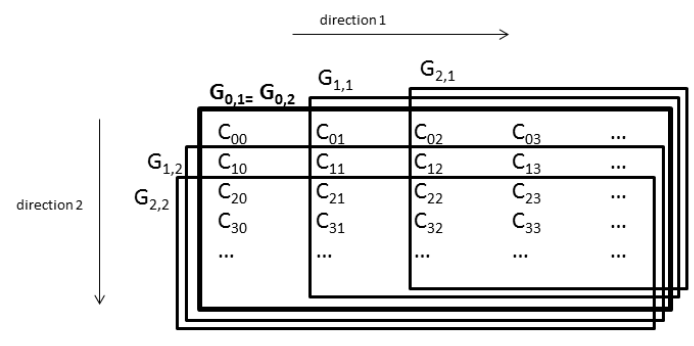

Fig. 1. Construction of the $\Omega_{21}$ and $\Omega_{22}$ sets of functions by simply shifting the Laguerre spectra independently along two directions.

Follows the definition of the error terms to be minimized:

$$
\begin{aligned}
& E_{21}\left(w_{1}, w_{2}\right)=\sum_{j=0}^{R_{21}} B_{j, 1} G_{j, 1}\left(w_{1}, w_{2}\right) \\
& E_{22}\left(w_{1}, w_{2}\right)=\sum_{j=0}^{R_{22}} B_{j, 2} G_{j, 2}\left(w_{1}, w_{2}\right)
\end{aligned}
$$

Setting $B_{R_{21}}=1$ and $B_{R_{22}}=1$ and independently solving two linear problems minimizing $\left\|E_{21}\right\|$ and $\left\|E_{22}\right\|$ yields denominators $D_{2,1}\left(w_{1}\right)$ and $D_{2,2}\left(w_{2}\right)$. Keeping (6) in mind the reduced order model is then constructed

$\bar{H}_{2}\left(w_{1}, w_{2}\right)=\frac{\sum_{i_{1}=0}^{R_{21}-1} \sum_{i_{2}=0}^{R_{22}-1} C_{i_{1} i_{2}} \sum_{j_{1}=1}^{R_{21}-i_{1}} B_{j_{1}+i_{1}, 1} w_{1}^{j_{1}} \sum_{j_{2}=1}^{R_{22}-i_{2}} B_{j_{2}+i_{2}, 2} w_{2}^{j_{2}}}{\sum_{j_{1}=0}^{R_{21}} B_{j_{1}, 1} w_{1}^{j_{1}} \sum_{j_{2}=0}^{R_{22}} B_{j_{2}, 2} w_{2}^{j_{2}}}$

The generalization to higher order kernels can easily be inferred when comparing (13) and (11). The method was purposefully designed to remain viable as the number of dimensions increases with each Volterra kernel.

It is important, at this stage, to clarify the notion of "order" and how the "order reduction" of a Volterra kernel will be subsequently quantified. For linear systems, a restrictive yet intuitive definition of model order reduction is the approximation of a rational transfer function by another rational transfer function of inferior order. The same is implied here albeit in a multilinear case. The order of the initial system is given by the truncation of the infinite series in (2). The order of the reduced system is given by the order of the multidimensional denominator in (6).

\section{Remarks}

While the compact format of the paper does not allow for every detail of the method to be illustrated and discussed, some issues should be noted.

- The choice of the free parameters $a_{m}$ is not trivial. An algorithm for selecting the parameters is given in [10]. It is a multilinear generalization of the results presented in [11] and [12].

- The numerators as defined by (11), (13) and their higher order generalizations were designed to preserve the Laguerre spectra of the original model to a certain extent. Thus for the first order kernel the first $R_{1}$ coefficients are preserved. For the second order kernel the first $R_{21} \times R_{22}$ coefficients are preserved and so on. In general, the numerator in equation (6) could be computed (for each kernel) by solving a linear means squares problem once the denominator is known. However as $m$ increases the problem quickly becomes large and ill conditioned. The suboptimal solution described above is simpler and more robust.

- For SISO systems it is reasonable to assume the kernels to be symmetrical, which simplifies to some extent the mathematical derivations above. However it was important for the authors to show that the method does handle non-symmetrical kernels in anticipation of future work on MIMO systems.

- Volterra models have polynomial static characteristics. The issue of accurately matching the static characteristics of the original system was discussed in [13]. Constraints could also be set on the reduced order model for increased DC accuracy however the authors considered this to be beyond the scope of this short paper.

\section{EXAMPLE AND SIMULATION RESULTS}

Theoretical papers on Volterra systems often use systems that are either both weakly nonlinear and have shallow dynamic effects. The authors designed a tunable test system inspired by the example in [14]. The LC filter with a nonlinear resistive load in [14] was completed by a non-adapted transmission line in order to introduce further dynamics. The load features a cubic nonlinearity and is described by the equation $i_{\text {out }}(t)=0.03 \cdot v_{\text {out }}(t)+0.02 \cdot v_{\text {out }}(t)^{2}+0.01 \cdot v_{\text {out }}(t)^{3}$.

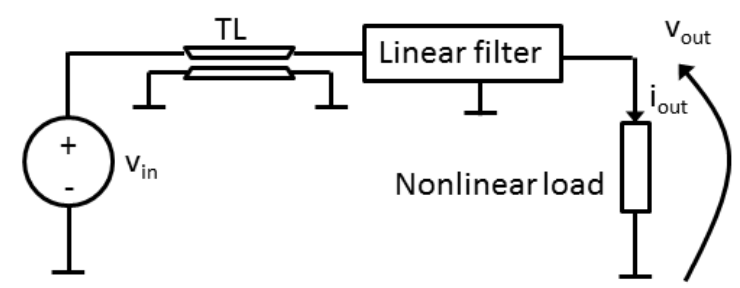

Fig. 2. Test setup: cascaded transmission line, linear filter and nonlinear load.

The system is driven by voltage generator $V_{i n}(t)$. 


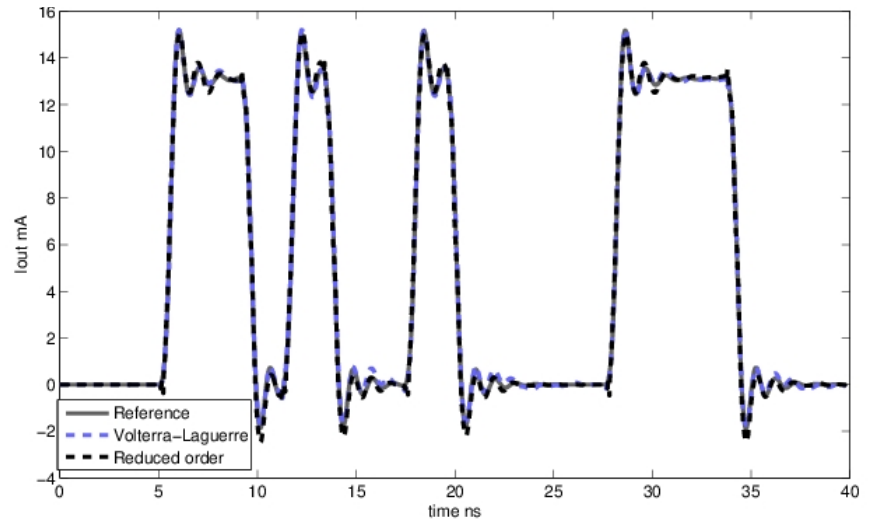

Fig. 3. Response of the test system (solid grey) its Volterra-Laguerre model (dashed blue) and its reduced order model (dashed black).

The relation binding the output current to the input voltage is modeled as a Volterra-Laguerre series with three kernels. After truncation, 38 coefficients were retained for the first kernel, $14 \times 14$ for the second kernel and $6 \times 6 \times 6$ for the third kernel. The detailed identification procedure is not within the main focus of the paper. Basically the system was implemented in SPICE and its output was observed for a rich training excitation exploring both the dynamic behavior of the system and its static characteristic between 0 and $1 \mathrm{~V}$. Following a stage of delay extraction, a simple means square method was used to compute the parameters. The accuracy of the identification was then tested using a validation signal. The three kernels were then approximated at orders $10,8 \times 8$ and $4 \times 4 \times 4$ respectively. The responses of the original system, the Volterra-Laguerre model and the reduced order model to the validation signal are shown in Fig. 3. The waveforms show a good match confirmed by error measurements. The normalized mean square error (NMSE) for the given waveform is $-35 \mathrm{~dB}$ for the Volterra-Laguerre model and $-30 \mathrm{~dB}$ for the much simpler reduced order model. Table 1 summarizes the data for an easier comparison. It's worth noting the gain in terms of accuracy/complexity ratio. Should a Volterra-Laguerre model be directly identified with the same number of parameters as the reduced order model the NMSE would increase to $-22 \mathrm{~dB}$.

\section{CONCLUSION}

In this proof-of-concept paper a method for approximating Volterra or Volterra-Laguerre kernels is presented. The concept is best described as multilinear model order reduction and seeks to gain speed-up by reducing parametric complexity while preserving accuracy. Initial tests such as the one presented in section IV show that the parametric complexity of the models can be drastically reduced. Since Volterra systems are used in various fields of science the technique may be a valuable tool for many users.
TABLE I. SimULATION RESULTS

\begin{tabular}{|l|c|c|c|c|}
\hline \multirow{2}{*}{\multicolumn{1}{c|}{ Model }} & \multicolumn{3}{|c|}{ Model order } & \multirow{2}{*}{ NMSE } \\
\cline { 2 - 4 } & $\boldsymbol{H}_{\boldsymbol{1}}$ & $\boldsymbol{H}_{\boldsymbol{2}}$ & $\boldsymbol{H}_{\mathbf{3}}$ & \\
\hline Volterra Laguerre & 38 & $14 \times 14=196$ & $6 \times 6 \times 6=216$ & $-35 \mathrm{~dB}$ \\
\hline Reduced order & 12 & $8 \times 8=64$ & $4 \times 4 \times 4=64$ & $-30 \mathrm{~dB}$ \\
\hline
\end{tabular}

\section{REFERENCES}

[1] V. Volterra, Theory of Functionals and of Integrals and IntegroDifferential Equations, Dover Publications, New York, 1959.

[2] N. Wiener, "Response of a nonlinear device to noise", Radiation Lab MIT 1942, restricted. report V-16, no 129 (112 pp). Declassified July 1946, Published as rep. no. PB-1-58087, U.S. Dept. Commerce.

[3] D. Jansson, A. Medvedev, "System identification of wiener systems via volterra-laguerre models: Application to human smooth pursuit analysis", European Control Conference (ECC), July 2015.

[4] X.Y.Z. Xiong, L.J. Jiang, J.E. Schutt-Ainé, W.C. Chew, "Volterra Series-Based Time-Domain Macromodeling of Nonlinear Circuits", IEEE Trans. on Components, Packaging and Manufacturing Technology, vol. 7, pp. 39-49, Jan. 2017.

[5] K. Batselier, Z. Chen, H. Liu, N. Wong "A tensor-based volterra series black-box nonlinear system identification and simulation framework", IEEE/ACM International Conference on Computer-Aided Design (ICCAD), Nov. 2016

[6] J.R. Phillips, "Projection-based approaches for model reduction of weakly nonlinear, time-varying systems", IEEE Trans. Comput.-Aided Design Integr. Circuits Syst., vol. 22, no. 2 , pp. 171 - 187, Feb. 2003.

[7] M. Rewienski and J. White, "A trajectory piecewise-linear approach to model order reduction and fast simulation of nonlinear circuits and micromachined devices", IEEE Trans. Comput.-Aided Design Integr. Circuits Syst., vol. 22, pp. 155 - 170, Feb. 2003.

[8] M. Telescu, N. Iassamen, P. Cloastre, N. Tanguy, “A simple algorithm for stable order reduction of z-domain Laguerre models" Signal Processing, Elsevier, vol. 13, pp.332-337, January 2013.

[9] D. Sidorov, "On Impulse Controle of Nonlinear Dynamical Systems Based on Volterra Series" in Integral Dynamical Models: Singularities, Signals and Control (World Scientific Series on Nonlinear Science, Series A, vol. 87), Singapore, World Scientific Publishing Co., 2015, ch. 11, sec. 11.2, pp 142 .

[10] A.Y. Kibangou, G. Favier, M. M. Hassani, "Laguerre-Volterra Filters Optimization Based on Laguerre Spectra,' Journal on Applied Signal Processing, vol. 17 , pp. 2874-2887, 2005.

[11] N. Tanguy, P. Vilbé, L.C. Calvez, "Optimum choice of free parameter in orthonormal approximations", IEEE Transactions on Automatic Control, vol. 40, pp. 1811-1813, Oct. 1995.

[12] N. Tanguy, R. Morvan, P. Vilbé, L.C. Calvez, "Online optimization of the time scale in adaptive Laguerre-based filters", IEEE Transactions on Signal Processing, pp. 1184-1187, vol. 48, Apr. 2000.

[13] C. Diouf, M. Telescu, P. Cloastre, N. Tanguy. "On the Use of Equality Constraints in the Identification of Volterra-Laguerre Models" IEEE Signal Processing Letters, vol. 19, pp. 857-860, Dec 2012.

[14] T. Wang, T.J. Brazil, "Volterra-mapping-based behavioral modeling of nonlinear circuits and systems for high frequencies", IEEE Transactions on Microwave Theory and Techniques, vol. 51, pp. 1433-1440, May 2003 . 